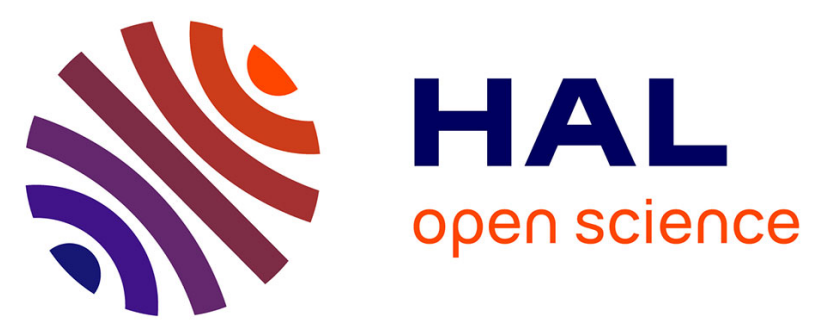

\title{
Human Respiratory Syncytial Virus-induced immune signature of infection revealed by transcriptome analysis of clinical pediatric nasopharyngeal swab samples
}

Claire Nicolas de Lamballerie, Andres Pizzorno, Julia Dubois, Blandine Padey, Thomas Julien, Aurélien Traversier, Julie Carbonneau, Elody Orcel, Bruno Lina, Marie-Ève Hamelin, et al.

\section{To cite this version:}

Claire Nicolas de Lamballerie, Andres Pizzorno, Julia Dubois, Blandine Padey, Thomas Julien, et al.. Human Respiratory Syncytial Virus-induced immune signature of infection revealed by transcriptome analysis of clinical pediatric nasopharyngeal swab samples. Journal of Infectious Diseases, In press, 223 (6), pp.1052-1061. 10.1093/infdis/jiaa468/5877957. hal-02909729

\section{HAL Id: hal-02909729 \\ https://hal.science/hal-02909729}

Submitted on 31 Jul 2020

HAL is a multi-disciplinary open access archive for the deposit and dissemination of scientific research documents, whether they are published or not. The documents may come from teaching and research institutions in France or abroad, or from public or private research centers.
L'archive ouverte pluridisciplinaire HAL, est destinée au dépôt et à la diffusion de documents scientifiques de niveau recherche, publiés ou non, émanant des établissements d'enseignement et de recherche français ou étrangers, des laboratoires publics ou privés. 


\section{Human Respiratory Syncytial Virus-induced immune}

signature of infection revealed by transcriptome analysis of clinical pediatric nasopharyngeal swab samples

Claire Nicolas de Lamballerie, Andrés Pizzorno, Julia Dubois, Blandine Padey, Thomas Julien, Aurélien Traversier, Julie Carbonneau, Elody Orcel, Bruno Lina, Marie-Eve Hamelin, Magali Roche, Julien Textoris, Guy Boivin, Catherine LegrasLachuer, Olivier Terrier, Manuel Rosa-Calatrava

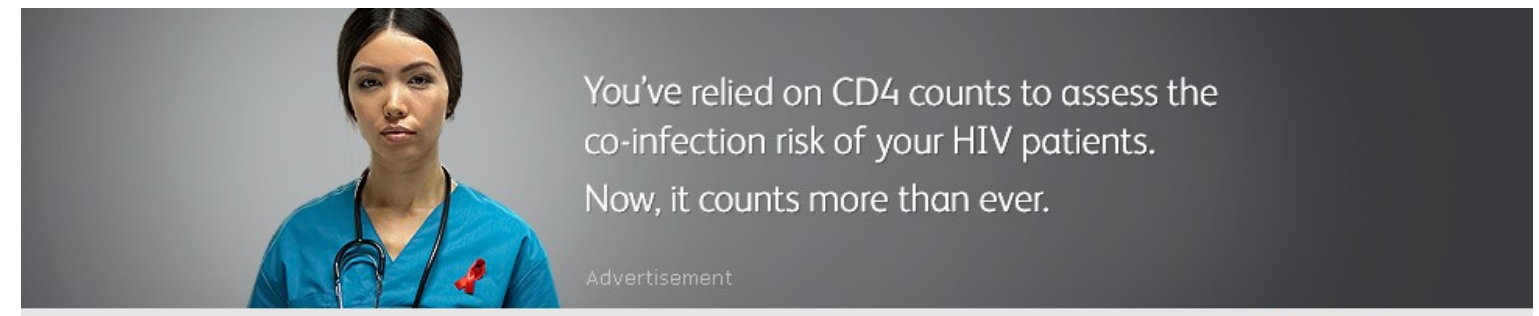




\section{Title}

Human Respiratory Syncytial Virus-induced immune signature of infection revealed by transcriptome analysis of clinical pediatric nasopharyngeal swab samples

\section{Authors}

Claire NICOLAS DE LAMBALLERIE ${ }^{1,2}$, Andrés PIZZORNO ${ }^{1}$, Julia DUBOIS ${ }^{1}$, Blandine PADEY $^{1}$, Thomas JULIEN ${ }^{1,3}$, Aurélien TRAVERSIER ${ }^{1}$, Julie CARBONNEAU ${ }^{4}$, Elody ORCEL $^{2}$, Bruno LINA ${ }^{1}$, Marie-Eve HAMELIN ${ }^{4}$, Magali ROCHE ${ }^{2}$, Julien TEXTORIS ${ }^{5}$, Guy BOIVIN $^{4}$, Catherine LEGRAS-LACHUER ${ }^{2,6}$, Olivier TERRIER ${ }^{1 \#,}$ Manuel ROSACALATRAVA ${ }^{1,3 \#}$

\#OT and MRC are co-last authors

\section{Affiliations}

1) CIRI, Centre International de Recherche en Infectiologie, (Team VirPath), Univ Lyon, Inserm, U1111, Université Claude Bernard Lyon 1, CNRS, UMR5308, ENS de Lyon, F69007, Lyon, France.

2) Viroscan3D SAS, Lyon 69008, France.

3) VirNext, Faculté de Médecine RTH Laennec, Université Claude Bernard Lyon 1, Université de Lyon, Lyon 69008, France.

4) Research Center in Infectious Diseases of the CHU de Quebec and Laval University, Quebec City, QC G1V 4G2, Canada.

5) Pathophysiology of Injury-Induced Immunosuppression (PI3), EA 7426 Hospices Civils de Lyon, bioMérieux, Université Claude Bernard Lyon 1, Hôpital Edouard Herriot, Lyon 69003, France. 
6) Ecologie Microbienne, UMR CNRS 5557, USC INRA 1364, Université Claude Bernard Lyon 1, Université de Lyon, Villeurbanne 69100, France.

\section{Corresponding authors}

olivier.terrier@univ-lyon1.fr; manuel.rosa-calatrava@univ-lyon1.fr

\section{Short summary}

In this study, Nicolas de Lamballerie \& colleagues applied a tailored transcriptomics workflow to exploit nasal wash samples from HRSV-positive hospitalized children. The transcriptional immune signature appears as a direct reflection of HRSV pathogenesis and highlights putative biomarkers of interest. 


\begin{abstract}
Human Respiratory Syncytial Virus (HRSV) constitutes one the main causes of respiratory infection in neonates and infants worldwide. Transcriptome analysis of clinical samples using high-throughput technologies remains an important tool to better understand virus-host complex interactions in the real-life setting but also to identify new diagnosis/prognosis markers or therapeutics targets. A major challenge when exploiting clinical samples such as nasal swabs, washes or bronchoalveolar lavages is the poor quantity and integrity of nucleic acids. In this study, we applied a tailored transcriptomics workflow to exploit nasal wash samples from children who tested positive for HRSV. Our analysis revealed a characteristic immune signature as a direct reflection of HRSV pathogenesis and highlighted putative biomarkers of interest such as IP-10, TMEM190, MCEMP1 or TIMM23.
\end{abstract}

\title{
Keywords
}

Respiratory Syncytial Virus; DNA microarray; Transcriptome; Nasal epithelium; NanoString assay 


\section{Background}

Respiratory Syncytial Virus (HRSV) constitutes one of the main causes of respiratory tract infection in newborns and young children worldwide [1] but also in the elderly, immunocompromised, and patients with chronic heart and lung conditions [2]. The global HRSV disease burden is estimated at approximately 200,000 deaths and more than 3 million hospitalizations per year [1,3]. Despite numerous attempts and ongoing clinical trials, no efficacious HRSV vaccine is yet available, and the specific therapeutic arsenal currently available is very limited and remains relatively expensive [4]. In this context, we urgently need to increase our understanding of HRSV pathogenesis and the multiple facets of its virus/host interactions.

Much of the HRSV-induced disease is considered as the reflection of the host innate immune response to infection $[5,6]$, with respiratory epithelial cells and monocytes/macrophages being the main actors in this response [7,8]. Indeed, HRSV infection was previously shown to upregulate the expression of host genes involved in the antiviral and cell-mediated immune responses, such as genes coding for interferons (IFNs) and more largely several cytokines/chemokines such as CXCL10/IP-10, CXCL8/IL-8, MCP-1/CCL2, RANTES/CCL5 or IL6 [8]. For example, we previously demonstrated that HRSV infection, alone or in the context of bacterial co-infection, strongly promotes CXCL10/IP-10 expression in human macrophages [9]. We also showed that HRSV infection of human respiratory epithelial cells induces a strong disequilibrium in the $\mathrm{p} 53 / \mathrm{NF}-\mathrm{kB}$ balance, which appears to contribute to the up-regulation of several proinflammatory cytokines and chemokines [10]. One limitation of these in vitro approaches is that they do not necessarily reflect the whole complexity of the in vivo environment. In this context, we advantageously investigated the HRSV-induced host response using an innovative and highly relevant primary human reconstituted airway epithelial 
model, cultivated at the air-liquid interface to assess previously undescribed facets of the HRSV biology, such as the impact of the infection on cilium mobility and morphogenesis [11].

The development of high-throughput "omics" approaches has contributed to deepen our understanding of the multiple levels of interplay between respiratory viruses and the host cell [12-15]. These approaches, in addition to be very informative about the dynamic interplay between the virus and the host and hence the pathogenesis mechanisms, could also constitute a powerful tool to identify new therapeutic targets and/or propose novel antiviral strategies. In the case of HRSV, few studies have investigated the transcriptomic host response using clinical specimens, and an even more limited number have exploited respiratory tract samples [16-18]. A major challenge associated with transcriptome analysis of clinical samples is the intrinsic low copy number and/or low integrity of the nucleic acids recovered. To tackle these hassles, several research groups, including ours, have proposed and developed adapted/optimized sample processes [19-21].

In this study, we investigated the impact of the infection on the host cell using nasal washes from hospitalized children with lab-confirmed HRSV infection. The analysis of HRSV-induced gene expression signature validated the importance of several IFN and cytokine-related pathways, in line with previous studies, but also provided valuable insight on potential biomarkers of diagnostic interest or as surrogates for the evaluation of future innovative treatments.

\section{Methods}

\section{Clinical samples and ethical considerations}

Written consent was obtained from parents of the three hospitalized children with labconfirmed HRSV infections. Control samples come from the collection of samples established 
by the Québec CHU in the context of RespiVir surveillance study. The protocol was approved by Ethics committee of the CHU de Québec-Université Laval. RNAs were obtained from nasal washes (in $1.5 \mathrm{~mL}$ saline) stored at $-80^{\circ} \mathrm{C}$. After thawing, the cell pellets were transferred to an RNAlater® Stabilization solution (Thermo Fisher Scientific).

\section{RNA extraction and microarray experiment}

Isolation of total RNA from nasal washes was performed using the RNeasy Micro kit (QIAGEN) with Dnase I treatment following the manufacturer's instructions. Samples were quantified using the Quantifluor RNA System (Promega) and qualified using Agilent RNA 6000 Pico chip on Bioanalyzer 2100 (Agilent Technologies). Whole RNA amplification using three rounds of in vitro transcription was then performed in two steps. First, the ExpressArt Trinucleotide mRNA amplification Pico kit (Amp Tec) was used for RNA amplification. Then, ss-cDNA synthesis was performed with the GeneChip® WT PLUS Reagent Kit (Affymetrix) with a minimal input requested of $5.5 \mu \mathrm{g}$. Labeled cRNA was hybridized to GeneChip Human Gene 2.0 ST Array (Affymetrix) for $16 \mathrm{~h}$ at $45^{\circ} \mathrm{C}$ and scanned using the Affymetrix $30007 \mathrm{G}$ Scanner. .CEL file generation and basic quality controls were performed with the GeneChip ${ }^{\mathrm{TM}}$ Command Console® (Affymetrix).

\section{Data analysis}

Data were analyzed using the R software and its xps (eXpression Profiling System) package (version 1.32.0) downloaded from www.bioconductor.org.. Quality controls were performed to assess technical bias, RNA degradation levels and background noise. Preprocessing steps consisted in background correction, Robust Multichip Average (RMA) normalization, probe summarization, and $\log 2$ transformation. A linear model was used to assess differential expression with the limma (Linear Models for Microarray Data) R/Bioconductor software 
package [22]. Genes were considered for subsequent analysis if they exhibited at least a 2-fold change in expression levels compared to the control samples coupled with p-values $<0.05$. In order to further functionally characterize the patient transcriptomic signature, the web-based tool DAVID 6.8 was used to determine the enriched pathways [23]. Genes predicted by TargetScan 7.2 [24] to be targeted by the up- or down- regulated miRNAs with cumulative weighted score $<-0.5$ were used for functional enrichment analysis using the same web-based tool. To further comprehend the connexions between the modulated genes in our study, we chose to represent the interactome as a graph where nodes correspond with proteins and edges with pairwise interactions using the web-based tool STRING 11.0 [30] (https://string-db.org), paired with Markov Clustering (MCL [26]) in order to extract relevant modules from such graphs.

\section{Pediatric mRNA datasets}

We chose three datasets from HRSV-infected host transcriptomic studies publicly available on Gene Expression Omnibus database (GEO) and ArrayExpress, based on peripheral pediatric blood samples (GSE69606 \& E-MTAB-5195) or PBMC gene expression responses to infection (GSE34205; n=51 HRSV-infected \& n=10 controls). We extracted raw data corresponding severe disease samples from the series GSE69606 (n=8) and MTAB-5195 $(n=18)$ and the recovery corresponding samples or healthy control samples (each $n=8$ ). Raw data were processed as previously described and differential analysis was performed according to the same thresholds ( $\mathrm{p}$-value $<0.05$ and absolute fold-change $>2$ ).

\section{Reconstituted human airway epithelia (HAE) and viruses}

MucilAir® human airway epithelia (HAE) model from Epithelix SARL was used for validation purposes. Infections (MOI of 1) were performed as previously described [11,21]. The Human 
Respiratory syncytial virus (HRSV-A Long strain ATCC-VR26) was produced in LLCMK2 cells (ATCC CCL7) in EMEM supplemented with $2 \mathrm{mM} \mathrm{L-glutamine} \mathrm{(Sigma} \mathrm{Aldrich),}$ penicillin $(100 \mathrm{U} / \mathrm{mL})$, streptomycin $(100 \mu \mathrm{g} / \mathrm{mL})\left(\right.$ Lonza), at $37{ }^{\circ} \mathrm{C}$ and $5 \% \mathrm{CO} 2$.

\section{NanoString nCounter validation}

The nCounter plateform (NanoString technologies) was used for mRNA detection of a 86 gene panel, according to manufacturer's instructions [27]. This custom panel gathers immunityrelated genes. Briefly, $300 \mathrm{ng}$ of total RNA were hybridized to the probes at $67^{\circ} \mathrm{C}$ for 18 hours using a thermocycler (Biometra, Tprofesssional TRIO, Analytik Jena AG, Jena, Germany). After loading into the nCounter Prep Station (NanoString Technologies) for purification and immobilization, the sample cartridge was then transferred and imaged on the nCounter Digital Analyzer (NanoString Technologies) Counts number were normalized by the geometric mean of HPRT1 (NM_000194.1), DECR1 (NM_001359.1), RPL19 (NM_000981.3), POLR2A (NM_000937.2) and TBP (NM_001172085.1) housekeeping genes count number, as well as the negative and positive control values using nSolver analysis software (version 4.0, NanoString technologies). Gene expression results are expressed in fold change induction compared to the mock-infected condition.

\section{Results}

\section{Differential gene expression in HRSV-infected samples}

In this study, we assessed nasal airway gene expression on pediatric nasal wash samples (3 infected and 5 controls). Given the low quality and low integrity of these sensitive samples (quality status available in Supplementary table 1), previously published adapted protocols were successfully used for their amplification and their subsequent hybridization on an 
Affymetrix GeneChip ${ }^{\mathrm{TM}}$ Human Gene 2.0ST [19]. An overview of the customized sample process and workflow is presented in Figure 1A and the subsequent hierarchical clustering of all analyzed samples is featured in Figure 1B. Despite the known heterogeneity of clinical samples, HRSV-infected and non-infected samples clustered appropriately to their corresponding experimental group.

For differential analysis, genes were considered significantly modulated if they exhibited at least a 2-fold change in expression levels compared to the control samples, with p-values inferior or equal to 0.05 . Using these criteria, we listed a total of 296 differentially expressed genes, $258(87.16 \%)$ of them being up-regulated (Supplementary table 2). This unbalanced up- versus down-regulated ratio was quite in line with previous observations [11]. As expected in the context of infected samples, many significantly up-regulated genes with fold changes far above 5 were related to the immune and IFN responses, such as ISG15, OASL, CXCL10/IP10, CCL2-3, IFITM1-3 or IRF1 (Supplementary table 2). In contrast, among the 38 downregulated genes, we listed genes associated with protein heterodimerization activity (SRGAP2C, HIST3H2BB and NTSR1), genes encoding zinc finger protein (ZNF439, ZNF28, ZNF286B, ZNF500) or transmembrane proteins acting as receptors or T-cell co-activators (such as SLC7A5P1, MSLNL or NTSR1). Of note, an important fraction (26\%) of the down-regulated genes was represented by miRNAs, such as hsa-miR-572, hsa-miR-486-2, hsa-miR-1229 or hsa-miR-663b (Supplementary table 2). Aside from miRNAs, only 3 other down-regulated genes (KIR3DL2, SLC7A5P1 and SRGAP2C) had fold changes lower than -3.

\section{Gene Ontology-based functional enrichment analysis}

To provide further functional interpretation of these clinical transcriptomic signatures, we then performed a Gene Ontology (GO)-based functional enrichment analysis using the web-based DAVID v6.8 toolkit (https://david.ncifcrf.gov/). GO terms, and particularly Biological 
Processes (BP), were considered enriched if their fold enrichment was higher than 2 and the Benjamini-Hochberg corrected enrichment p-value was inferior to 0.05. This BP enrichment was based on the global list of deregulated genes (Figure 2). As anticipated, the most enriched BP were primarily associated with interferon response (ex: GO:0060337; GO:0060333), response to virus (ex: GO:0009615; GO:0051607) or antigen processing (ex: GO:0002479; GO:0042612), which represent 16 out of the 24 most enriched BP listed (Figure 2A). Interestingly, the remaining GO terms were mainly related to mitochondria/respiratory burst (ex: GO:0005739; GO:0045730; GO:0045454) or ubiquitin ligase (ex: GO:0051437; GO:0051436).

To better illustrate the impact of HRSV infection on the host immunity-related genes, we used the list of up-regulated genes and explored the functional association networks of their protein products using the STRING [25] database (https://string-db.org). As presented in Figure 2B, this analysis highlighted a functional network based on 241 distinct proteins (nodes) and 637 protein-protein associations (edges). These associations, which highlight proteins sharing functions but not necessarily physical interaction, are categorized into 15 relevant clusters, among which 10 contained more than 3 proteins (each color $=1$ cluster by Markov Clustering [26] or MCL). Two major hubs concentrating a large number of edges were identified. The main hub consisted of proteins related to the immune response, with a central place for major actors like CXCL10, OASL and ISG15 (Figure 2B, red dots). The second hub harbored proteins involved in the positive and negative regulation of ubiquitin-protein ligase activity during the mitotic cell cycle (GO:0051436 and GO:0051437, medium purple dots). Much as the first hub would have been expected in an infectious context, the specific modulation of genes related to respiratory burst, cell redox homeostasis or ubiquitin-protein ligase activity by HRSV infection has been less studied. 
Because of the numerous miRNAs deregulated, we used a prediction algorithm (TargetScan 7.2 [24]) for the identification of all genes targeted by the down- and up- regulated miRNAs. The predicted genes targeted by the down-regulated miRNAs were related to biological processes such as phagocytosis (GO:0006909), peptide cross-linking (GO:0018149) or positive regulation of release of cytochrome c from mitochondria (GO:0090200). Interestingly, the predicted targets of the up-regulated miRNAs are linked to similar processes: negative regulation of nucleic acid-templated transcription (GO:1903507) and negative regulation of T cell proliferation (GO:0042130) for the most enriched BPs (Supplementary Figure 1).

\section{Tissue-specific gene expression}

In order to describe tissue-specific HRSV infection signatures, differentially expressed gene lists were extracted from three pediatric mRNA array datasets [28-30] and compared to our data to highlight similarities and differences between blood and respiratory airway transcriptional profiles, respectively (Figure 3). As previously shown, the overlap between the blood/PBMC and respiratory tract gene expression is scarce [29]. Only 6 genes (ANXA3, FCGR1B, OASL, BCL2A1, CLEC4D, RSAD2) were upregulated in all analyzed datasets, mostly associated with the host immune response to the infection. As expected, both studies on peripheral blood shared a specific signature composed of 228 genes, whereas 51 additional genes were also modulated in PBMCs. These genes are mostly associated with the innate immune response of the host (GO:0045087). When comparing these signatures with the list of genes deregulated in our study, we highlighted 242 genes exclusively modulated in nasal washes, hence constituting specific drivers of the nasal epithelium signature. Among these tissue-specific modulated genes, genes were associated with the immune response regulation (GO:0050776) and, more precisely, with type I interferon signaling pathway (GO:0060337), or antigen processing and presentation (GO:0002479). 


\section{Validation of differential expression in reconstituted human airway epithelium (HAE)}

We then sought to validate these results in the context of experimental infections with the prototype HRSV A Long strain $(\mathrm{MOI}=1)$ in a human reconstituted airway epithelial $(\mathrm{HAE})$ model, as previously described [11]. This HAE model, issued from healthy donor biopsies, is composed of human primary ciliated columnar cells, mucus-secreting goblet and basal cells cultivated at the air-liquid interface, and has been successfully used to study viral infections in previous studies [11,21]. After 6 days of infection, HAE were lysed and total RNA was extracted and subsequently analyzed with the NanoString nCounter platform using a customized 94 immunity-related (cytokine production, T cell proliferation, interferon-gammamediated signaling pathway, etc) gene panel [27]. As shown in Figure 4, 39 out of the 94 genes in the NanoString panel were differentially modulated in the HRSV-infected condition compared to the mock-infected control. Despite the differential nature of infectious samples, the comparison of global gene expression modulation results between Affymetrix microarray (clinical samples) and NanoString (experimental infections in HAE) assays showed a correlation coefficient of 0.63 . This relatively high correlation could be explained by the fact that both types of samples contain cells of nasal origin. Unsurprisingly, the most up-regulated gene in the infectious context is CXCL10/IP-10, followed by IFI44L, IDO1 and TNFSF13B, with expression ratios above 50 (Figure 4). The top 20 modulated genes are strongly linked to "type I interferon signaling pathway" (GO:0060337) or more widely to "response to virus" (GO:0009615 or GO:0051607). Altogether, the gene expression results observed in clinical samples were cross-validated using an alternative method and underline a global deregulation of the biological defenses of the host, notably in the case of interferon stimulated genes (ISGs) that constitute a hallmark of many infectious and/or autoimmune disease states. 


\section{Discussion}

In the particular context of HRSV infections, most of the respiratory samples collected for clinical studies are issued from pediatric patients, from whom only limited amounts of material are obtained. Moreover, given the fact that the main purpose of patient sampling is usually clinical analysis rather than research, the low quantity and quality of exploitable material is far from being optimal. In this study, we showed that these hurdles for the exploitation of highly degraded clinical samples could be mitigated by using adapted protocols and microarray, such as the Affymetrix GeneChip Human Gene 2.0 ST. Thus, despite starting from a limited number of children nasal washes presenting an acute HRSV infection, our adapted sample-processing pipeline enabled the determination and characterization of robust pediatric HRSV-induced nasal transcriptome signatures. In this study, patient-derived samples were processed with adapted protocols and transcriptomic signatures were obtained by hybridization on the HuGene 2.0 st Affymetrix microarray and subsequent process of the data. We compared our results to published pediatrics blood microarray datasets for the establishment of a nasal-specific signature. We also included biological results obtained using our previously described relevant human reconstituted airway epithelial (HAE) model of HRSV infection [11] for a deeper comprehension of the virus impact on the host epithelium.

Initial signature analysis enabled the identification of well known markers of HRSV infection, namely the upheaval of the immune cascade [15-17], notably highlighting the strong overturning of CXCL10/IP-10 gene expression. We then advantageously used a biologically relevant reconstituted human airway epithelia (HAE) model to reproduce and validate these results by NanoString nCounter assay. IP-10 has already been described as the most abundant cytokine in bronchoalveolar lavages collected from HRSV-infected patients [31]. We also identified up-regulated genes seemingly commonly modulated in different infection scenarios. For example, HRASLS2 is highly induced in RV infection [32], FFAR2 promotes 
internalization during IAV entry [33], and IFI6 has pleiotropic functions in HRSV, Dengue, or hepatitis C virus infections [34-36]. Conversely, our analysis revealed TMEM190 and MCEMP1 as potential specific biomarkers of HRSV infection. Although, TMEM190 expression was largely decreased in small airway epithelium by smoking [37] and MCEMP1 was proposed as a biomarker for stroke prognosis [38], a particular expression profile of these genes has not yet been described on other viral infections, for which they underscore further study as potential biomarkers. A similar rationale supports the study of TIMM23, strongly upregulated in our study. A previous study conducted by Zaas et al. [15] identified a panel of 15 genes specifically modulated in HRSV-infected adults, of which FCGR1B, GBP1, RTP4, RSAD2, ISG15, IFIT2 were also significantly deregulated in our study. Despite the different nature of the biological samples used (children nasal washes versus adult blood), the high degree of concordance observed between our results and theirs supports a distinctive HRSV infection signature.

In addition to genes related to the immune response, our transcriptomics results also highlighted the modulation of genes and pathways related to a global mitochondrion cellular process disruption. This suggests that HRSV infection could unsettle less described biological processes related to the cAMP cascade, the redox complexes of mitochondrial respiratory chain (respiratory burst). Although, this impact on the respiratory chain is a less explored aspect of HRSV pathogenesis in the respiratory tract, a study from Bataki et al. [39] investigated whether HRSV can directly signal to activate neutrophil cytotoxic function or not in the context of infant bronchiolitis. Besides HRSV infections, the respiratory burst is known to be defective in influenza-infected neutrophils or during co-infections [40,41]. The disruption of such metabolic process could be a first clue regarding prognostic evolutions of children infected by HRSV.

In addition, the biological interpretation of the 38 down-regulated genes is not as straightforward. Indeed, no biological process or function was significantly enriched in our 
study, only modulations of individual gene were highlighted. Some markers, already described in the context of other viral infections, were found in the top down-regulated genes $[42,43]$. This protection is known to be mediated partially by the inhibitory NK cell receptor KIR3DL2, whose gene expression is strongly inhibited in our HRSV infectious context. The SLC7A5P1 gene is also predicted to be linked to NK cells (GO:0032825 positive regulation of natural killer cell differentiation) whereas SRGAP2C had already be linked to HRSV bronchiolitis. Interestingly, we also observed the significant downregulation of several miRNAs. Among them, some are already described in literature such as miRNA-572, prognosis biomarker for renal cell carcinoma and sclerosis $[44,45]$, or miRNA-769, included in a miRNA panel for discrimination between Mycobacterium tuberculosis infected and healthy individuals [46].

Regardless of the studied tissue, HRSV is consensually described as a major disruptor of the host immune response [47-49]. Here, comparing our signatures with 3 other ones extracted from pediatric whole blood transcriptomic analyses [28-30], we highlighted the common deregulation of 7 genes, independently of the tissue, and interestingly, 242 genes that seem to be specific to nasal epithelium HRSV-induced gene expression, in line with data from literature $[50]$.

Collectively, the transcriptomic analysis of nasal wash samples highlights the qualitative importance of such clinical samples, particularly in the context of their limited availability. The results obtained with a complementary approach such as the reconstituted HAE greatly contribute to bridge the knowledge gap in the understanding of the specific effects of HRSV on the host respiratory tissue and pave the way for several so far undescribed avenues of investigation. 


\section{Acknowledgements \& Funding sources}

The authors want to thank Sophie Assant for her help with the NanoString nCounter assay and Epithelix (Switzerland) for its help with MucilAir® human airway epithelia (HAE). This work was funded by grants from Région Auvergne Rhône-Alpes (CMIRA N ${ }^{\circ} 14007029$ and AccueilPro COOPERA $\mathrm{N}^{\circ} 15458$ grants), and Canadian Institutes of Health Research $\left(\mathrm{N}^{\circ}\right.$ 229733 and 230187). Claire Nicolas de Lamballerie was funded by National Association for Research in Technology (ANRT). Guy Boivin is the holder of the Canada Research Chair on influenza and other respiratory viruses. Funding institutions had no participation in the design of the study, collection, analysis and interpretation of data, or in the writing of the manuscript. The authors declare they have no competing interests.

\section{Author contributions}

CNDL, AP, BL, GB, CLL, OT, MRC participated to conception and coordination of the study. CNDL, AP, BP, JC, EO, TJ, AT, BL, MEH, MR, JT, GB, CLL, OT, MRC carried out the experiments and analysis of the results. CNDL, JD, OT, AP, MRC designed the study and wrote the manuscript. 


\section{References}

1. Nair H, Nokes DJ, Gessner BD, et al. Global burden of acute lower respiratory infections due to respiratory syncytial virus in young children: a systematic review and meta-analysis. Lancet [Internet]. 2010; 375(9725):1545-1555. Available from: https://linkinghub.elsevier.com/retrieve/pii/S0140673610602061

2. Falsey AR, Hennessey PA, Formica MA, Cox C, Walsh EE. Respiratory Syncytial Virus Infection in Elderly and High-Risk Adults. N Engl J Med [Internet]. Massachusetts Medical Society ; 2005 [cited 2019 Mar 26]; 352(17):1749-1759. Available from: http://www.nejm.org/doi/abs/10.1056/NEJMoa043951

3. Hall CB, Simőes EAF, Anderson LJ. Clinical and Epidemiologic Features of Respiratory Syncytial Virus. Springer, Berlin, Heidelberg; 2013 [cited 2019 Nov 7]. p. 39-57. Available from: http://link.springer.com/10.1007/978-3-642-38919-1_2

4. Griffiths C, Drews SJ, Marchant DJ. Respiratory Syncytial Virus: Infection, Detection, and New Options for Prevention and Treatment. Clin Microbiol Rev [Internet]. American Society for Microbiology (ASM); 2017 [cited 2019 Nov 7]; 30(1):277-319. Available from: http://www.ncbi.nlm.nih.gov/pubmed/27903593

5. Durbin JE, Johnson TR, Durbin RK, et al. The role of IFN in respiratory syncytial virus pathogenesis. J Immunol [Internet]. American Association of Immunologists; 2002 [cited 2019 Nov 7]; 168(6):2944-52. Available from: http://www.ncbi.nlm.nih.gov/pubmed/8345194

6. Ramaswamy M, Shi L, Monick MM, Hunninghake GW, Look DC. Specific Inhibition of Type I Interferon Signal Transduction by Respiratory Syncytial Virus. Am J Respir Cell Mol Biol [Internet]. American Thoracic Society; 2004 [cited 2019 Nov 7]; 30(6):893-900. Available from: http://www.atsjournals.org/doi/abs/10.1165/rcmb.2003-0410OC 
7. Tripp RA, Oshansky C, Alvarez R. Cytokines and Respiratory Syncytial Virus Infection. Proc Am Thorac Soc [Internet]. 2005 [cited 2019 Nov 7]; 2(2):147-149. Available from: http://pats.atsjournals.org/cgi/doi/10.1513/pats.200502-014AW

8. Russell CD, Unger SA, Walton M, Schwarze J. The Human Immune Response to Respiratory Syncytial Virus Infection. Clin Microbiol Rev [Internet]. American Society for Microbiology (ASM); 2017 [cited 2019 Nov 7]; 30(2):481-502. Available from: http://www.ncbi.nlm.nih.gov/pubmed/28179378

9. Machado D, Hoffmann J, Moroso M, et al. RSV Infection in Human Macrophages Promotes CXCL10/IP-10 Expression during Bacterial Co-Infection. Int J Mol Sci [Internet]. Multidisciplinary Digital Publishing Institute; 2017 [cited 2019 Mar 22]; 18(12):2654. Available from: http://www.mdpi.com/1422-0067/18/12/2654

10. Machado D, Pizzorno A, Hoffmann J, et al. Role of p53/NF- $\kappa B$ functional balance in respiratory syncytial virus-induced inflammation response. J Gen Virol [Internet]. Microbiology Society; 2018 [cited 2019 Nov 7]; 99(4):489-500. Available from: https://www.microbiologyresearch.org/content/journal/jgv/10.1099/jgv.0.001040

11. Nicolas de Lamballerie C, Pizzorno A, Dubois J, et al. Characterization of cellular transcriptomic signatures induced by different respiratory viruses in human reconstituted airway epithelia. Sci Rep [Internet]. Nature Publishing Group; 2019 [cited 2019 Nov 7]; 9(1):11493. Available from: http://www.ncbi.nlm.nih.gov/pubmed/31391513

12. Parnell GP, McLean AS, Booth DR, et al. A distinct influenza infection signature in the blood transcriptome of patients with severe community-acquired pneumonia. Crit Care [Internet]. BioMed Central; 2012 [cited 2019 Nov 7]; 16(4):R157. Available from: http://www.ncbi.nlm.nih.gov/pubmed/22898401

13. Hancock AS, Stairiker CJ, Boesteanu AC, et al. Transcriptome Analysis of Infected 
and Bystander Type 2 Alveolar Epithelial Cells during Influenza A Virus Infection Reveals In Vivo Wnt Pathway Downregulation. J Virol [Internet]. American Society for Microbiology (ASM); 2018 [cited 2019 Nov 7]; 92(21). Available from: http://www.ncbi.nlm.nih.gov/pubmed/30111569

14. Troy NM, Bosco A. Respiratory viral infections and host responses; insights from genomics. Respir Res. 2016; 17(1):1-12.

15. Zaas AK, Chen M, Varkey J, et al. Gene Expression Signatures Diagnose Influenza and Other Symptomatic Respiratory Viral Infection in Humans. Cell Host Microbe. 2009; 6(3):207-217.

16. Levitz R, Gao Y, Dozmorov I, Song R, Wakeland EK, Kahn JS. Distinct patterns of innate immune activation by clinical isolates of respiratory syncytial virus. PLoS One [Internet]. Public Library of Science; 2017 [cited 2019 Nov 7]; 12(9):e0184318. Available from: http://www.ncbi.nlm.nih.gov/pubmed/28877226

17. Do LAH, Pellet J, Doorn HR van, et al. Host Transcription Profile in Nasal Epithelium and Whole Blood of Hospitalized Children Under 2 Years of Age With Respiratory Syncytial Virus Infection. J Infect Dis [Internet]. Oxford University Press; 2017 [cited 2019 Nov 5]; 217(1):134-146. Available from: http://www.ncbi.nlm.nih.gov/pubmed/29029245

18. Ampuero S, Andaur R, Milano M, et al. Time-course of transcriptome response to respiratory syncytial virus infection in lung epithelium cells. Acta Virol [Internet]. 2018 [cited 2019 Nov 7]; 62(03):310-325. Available from: http://www.elis.sk/index.php?page=shop.product_details\&flypage=flypage.tpl\&produc t_id=5804\&category_id=145\&option=com_virtuemart

19. Dupinay T, Nguyen A, Croze S, et al. Next-generation sequencing of ultra-low copy samples : from clinical FFPE samples to single-cell sequencing. Curr Top Virol 
[Internet]. 2012 [cited 2019 Mar 21]; 10(June 2015):63-83. Available from: https://www.researchgate.net/publication/278684713_Nextgeneration_sequencing_of_ultralow_copy_samples_from_clinical_FFPE_samples_to_single-cell_sequencing

20. Degletagne C, Keime C, Rey B, et al. Transcriptome analysis in non-model species: a new method for the analysis of heterologous hybridization on microarrays. BMC Genomics [Internet]. BioMed Central; 2010 [cited 2019 Mar 21]; 11:344. Available from: http://www.ncbi.nlm.nih.gov/pubmed/20509979

21. Pizzorno A, Terrier O, Nicolas de Lamballerie C, et al. Repurposing of Drugs as Novel Influenza Inhibitors From Clinical Gene Expression Infection Signatures. Front Immunol [Internet]. Frontiers; 2019 [cited 2019 Mar 22]; 10:60. Available from: https://www.frontiersin.org/article/10.3389/fimmu.2019.00060/full

22. Ritchie ME, Phipson B, Wu D, et al. limma powers differential expression analyses for RNA-sequencing and microarray studies. Nucleic Acids Res [Internet]. 2015 [cited 2017 Nov 14]; 43(7). Available from: https://www.ncbi.nlm.nih.gov/pmc/articles/PMC4402510/pdf/gkv007.pdf

23. Dennis G, Sherman BT, Hosack DA, et al. DAVID: Database for Annotation, Visualization, and Integrated Discovery. Genome Biol [Internet]. 2003 [cited 2019 Mar 21]; 4(5):P3. Available from: http://www.ncbi.nlm.nih.gov/pubmed/12734009

24. Lewis BP, Shih IH, Jones-Rhoades MW, Bartel DP, Burge CB. Prediction of Mammalian MicroRNA Targets. Cell. Cell Press; 2003; 115(7):787-798.

25. Szklarczyk D, Franceschini A, Wyder S, et al. STRING v10: protein-protein interaction networks, integrated over the tree of life. Nucleic Acids Res [Internet]. 2015 [cited 2019 Mar 21]; 43(D1):D447-D452. Available from: http://www.ncbi.nlm.nih.gov/pubmed/25352553 
26. S. VD. Graph clustering by flow simulation. 2000.

27. Tsang H-F, Xue VW, Koh S-P, Chiu Y-M, Ng LP-W, Wong S-CC. NanoString, a novel digital color-coded barcode technology: current and future applications in molecular diagnostics. Expert Rev Mol Diagn [Internet]. 2017 [cited 2019 Apr 10]; 17(1):95-103. Available from: http://www.ncbi.nlm.nih.gov/pubmed/27917695

28. Jong VL, Ahout IML, Ham H-J Van Den, et al. Transcriptome assists prognosis of disease severity in respiratory syncytial virus infected infants. Nat Publ Gr. 2016; .

29. Ioannidis I, McNally B, Willette M, et al. Plasticity and Virus Specificity of the Airway Epithelial Cell Immune Response during Respiratory Virus Infection. J Virol. American Society for Microbiology; 2012; 86(10):5422-5436.

30. Brand HK, Ahout IML, Ridder D De, et al. Olfactomedin 4 serves as a marker for disease severity in pediatric respiratory syncytial virus (RSV) infection. PLoS One. Public Library of Science; 2015; 10(7).

31. McNamara PS, Flanagan BF, Hart CA, Smyth RL. Production of Chemokines in the Lungs of Infants with Severe Respiratory Syncytial Virus Bronchiolitis. J Infect Dis [Internet]. 2005 [cited 2019 Nov 13]; 191(8):1225-1232. Available from: https://academic.oup.com/jid/article-lookup/doi/10.1086/428855

32. Chen Y, Hamati E, Lee PK, et al. Rhinovirus induces airway epithelial gene expression through double-stranded RNA and IFN-dependent pathways. Am J Respir Cell Mol Biol. American Thoracic Society; 2006; 34(2):192-203.

33. Wang G, Jiang L, Wang J, et al. The G Protein-Coupled Receptor FFAR2 Promotes Internalization during Influenza A Virus Entry. J Virol. American Society for Microbiology; 2019; 94(2).

34. Mejias A, Dimo B, Suarez NM, et al. Whole Blood Gene Expression Profiles to Assess Pathogenesis and Disease Severity in Infants with Respiratory Syncytial Virus 
Infection. PLoS Med. 2013; 10(11).

35. Qi Y, Li Y, Zhang Y, et al. IFI6 Inhibits Apoptosis via Mitochondrial-Dependent Pathway in Dengue Virus 2 Infected Vascular Endothelial Cells. Jin X, editor. PLoS

One [Internet]. Public Library of Science; 2015 [cited 2020 Apr 15]; 10(8):e0132743. Available from: https://dx.plos.org/10.1371/journal.pone.0132743

36. Meyer K, Kwon YC, Liu S, Hagedorn CH, Ray RB, Ray R. Interferon- $\alpha$ inducible protein 6 impairs EGFR activation by CD81 and inhibits hepatitis C virus infection. Sci Rep. Nature Publishing Group; 2015; 5(1):1-10.

37. Hackett NR, Butler MW, Shaykhiev R, et al. RNA-Seq quantification of the human small airway epithelium transcriptome. BMC Genomics. BioMed Central; 2012; 13(1):82.

38. Raman K, O’Donnell MJ, Czlonkowska A, et al. Peripheral Blood MCEMP1 Gene Expression as a Biomarker for Stroke Prognosis. Stroke. Lippincott Williams and Wilkins; 2016; 47(3):652-658.

39. Bataki EL, Evans GS, Everard ML. Respiratory syncytial virus and neutrophil activation. Clin Exp Immunol. 2005; 140(3):470-477.

40. Heo JY, Song JY, Noh JY, et al. Effects of influenza immunization on pneumonia in the elderly. Hum. Vaccines Immunother. Taylor and Francis Inc.; 2018. p. 744-749.

41. Bosch AATM, Biesbroek G, Trzcinski K, Sanders EAM, Bogaert D. Viral and Bacterial Interactions in the Upper Respiratory Tract. PLoS Pathog. Public Library of Science; 2013.

42. Hansasuta P, Dong T, Thananchai H, et al. Recognition of HLA-A3 and HLA-A11 by KIR3DL2 is peptide-specific. Eur J Immunol. 2004; 34(6):1673-1679.

43. Cosman D, Fanger N, Borges L, et al. A novel immunoglobulin superfamily receptor for cellular and viral MHC class I molecules. Immunity. Cell Press; 1997; 7(2):273- 
282.

44. Pan X, Li Z, Zhao L, et al. microRNA-572 functions as an oncogene and a potential biomarker for renal cell carcinoma prognosis. Oncol Rep [Internet]. Spandidos Publications; 2018 [cited 2020 Apr 15]; 40(5):3092-3101. Available from: http://www.ncbi.nlm.nih.gov/pubmed/30132566

45. Mancuso R, Hernis A, Agostini S, Rovaris M, Caputo D, Clerici M. MicroRNA-572 expression in multiple sclerosis patients with different patterns of clinical progression. J Transl Med [Internet]. BioMed Central Ltd.; 2015 [cited 2020 Apr 15]; 13(1):148. Available from: http://www.translational-medicine.com/content/13/1/148

46. Cui JY, Liang HW, Pan XL, et al. Characterization of a novel panel of plasma microRNAs that discriminates between Mycobacterium tuberculosis infection and healthy individuals. PLoS One. Public Library of Science; 2017; 12(9).

47. Hijano DR, Vu LD, Kauvar LM, Tripp RA, Polack FP, Cormier SA. Role of Type I Interferon (IFN) in the Respiratory Syncytial Virus (RSV) Immune Response and Disease Severity. Front Immunol [Internet]. Frontiers; 2019 [cited 2019 Jun 6]; 10:566. Available from: https://www.frontiersin.org/article/10.3389/fimmu.2019.00566/full

48. Heinonen S, Rodriguez-Fernandez R, Diaz A, Rodriguez-Pastor SO, Ramilo O, Mejias A. Infant Immune Response to Respiratory Viral Infections. Immunol. Allergy Clin. North Am. W.B. Saunders; 2019. p. 361-376.

49. Carvajal JJ, Avellaneda AM, Salazar-Ardiles C, Maya JE, Kalergis AM, Lay MK. Host Components Contributing to Respiratory Syncytial Virus Pathogenesis. Front. Immunol. Frontiers Media S.A.; 2019. p. 2152.

50. Pennings JLA, Schuurhof A, Hodemaekers HM, et al. Systemic Signature of the Lung Response to Respiratory Syncytial Virus Infection. Ahuja SK, editor. PLoS One [Internet]. Public Library of Science; 2011 [cited 2020 Apr 6]; 6(6):e21461. Available 
from: https://dx.plos.org/10.1371/journal.pone.0021461 


\section{Figure legends}

Figure 1. Sample processing workflow and transcriptomic hierarchical clustering. (A) Adapted workflow for the processing and exploitation of clinical samples with low RNA quality/quantity. (B) Hierarchical clustering of the signal intensities corresponding to all infected and non-infected clinical samples evaluated in the study. The resulting clusters are representative of the degree of similarity between samples and enable clustering into infected and non-infected experimental groups. The height of the y-axis at the branching points is a measure of similarity; y-axis units are arbitrary. For representation purposes, data was autoscaled and $\log 2$ transformed.

\section{Figure 2. Gene Ontology-based functional enrichment and protein-protein interaction} analyses. (A) Enriched biological process terms corresponding to the up-regulated gene list (enrichment score $>2$ and Benjamini-Hochberg corrected p-value $<0.05$ ). The down-regulated gene list did not present sufficient enrichment to pass our thresholds. (B) Evidence view of predicted protein associations associated with up-regulated genes in the HRSV-infected condition. Network nodes are host proteins and edges represent predicted functional associations. The color-coded lines correspond to the types of evidence supporting predicted associations (minimum required interaction score: 0.7). Node colors correspond to Markov clusters (MCL, inflation parameter: 1.5).

Figure 3. Gene expression cross-analysis as a key to tissue-specific local reaction to infection. (A) We selected 3 pediatric mRNA expression datasets for gene expression comparison across tissues. The GSE69606 dataset combines samples from 26 patients with acute HRSV infections, with symptoms spanning from mild to severe and the corresponding recovery paired samples. The E-MTAB-5195 dataset was originally used to investigate blood 
transcriptomics of 39 children during HRSV infection and for a longitudinal analysis to determine an 84-gene prognosis signature discriminating hospitalized infants with severe HRSV disease from infants with mild symptoms. The GSE34205 dataset is part of a wider study (GSE32140), aiming at establishing the signature induced by influenza and HRSV on PBMCs and primary airway epithelial cells. Because of the disease status of our study samples, we focused on severely ill children infected by HRSV. (B) Comparative cross-analysis of the gene lists on the 4 datasets ( 3 external plus ours). Common and specific infection features and gene enrichment analysis applied to the 242 nasal-specific genes are shown.

Figure 4. Experimental validation of gene expression results by NanoString assay in human airway epithelia (HAE). The expression of immunity-related genes in infected nasal HAE was validated using Nanostring nCounter technology. Data processing and normalization were performed with nSolver 4.0 analysis software and significant results (absolute fold change > 2) are expressed in fold change induction compared to the mock-infected condition. 
Figure 1
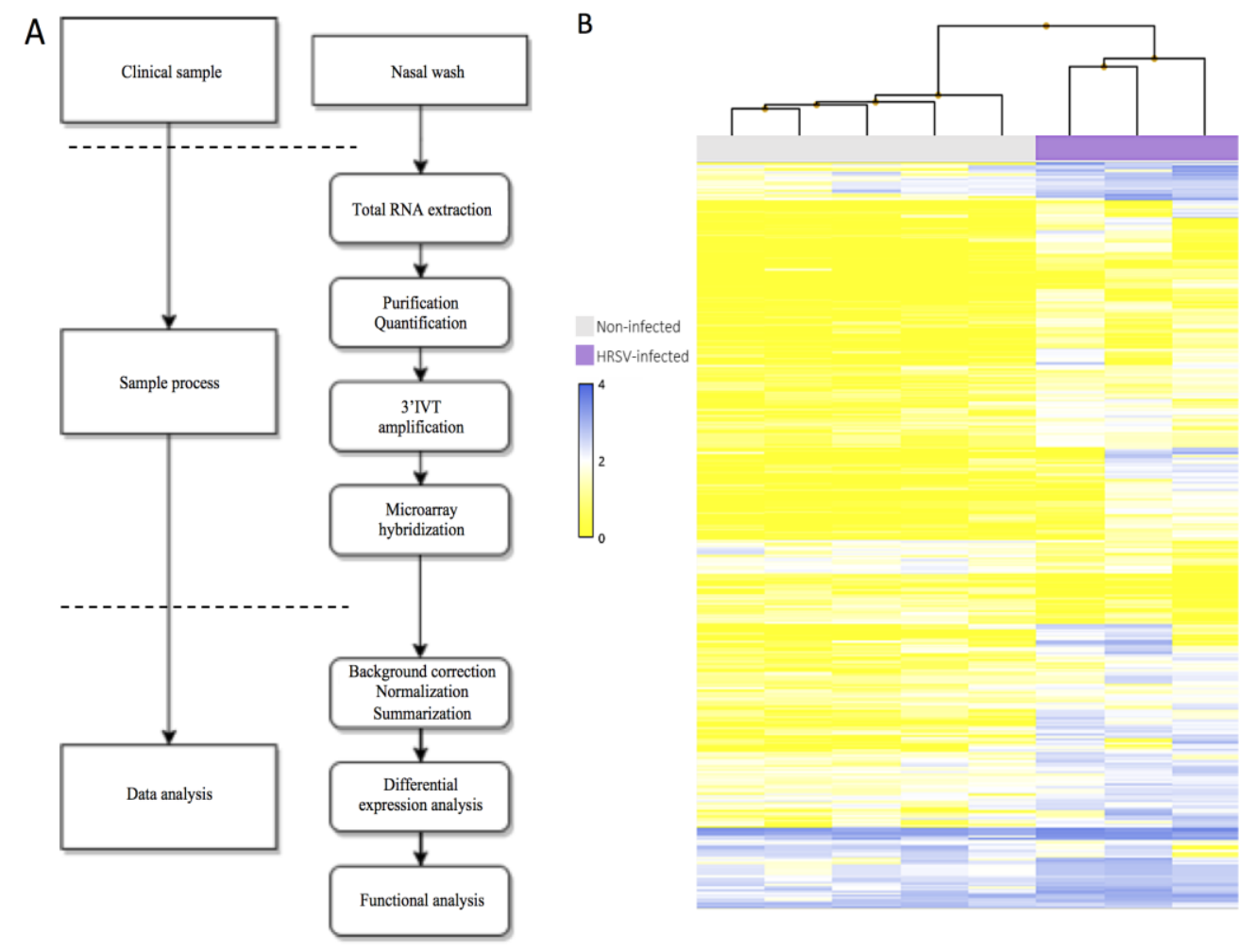
Figure 2

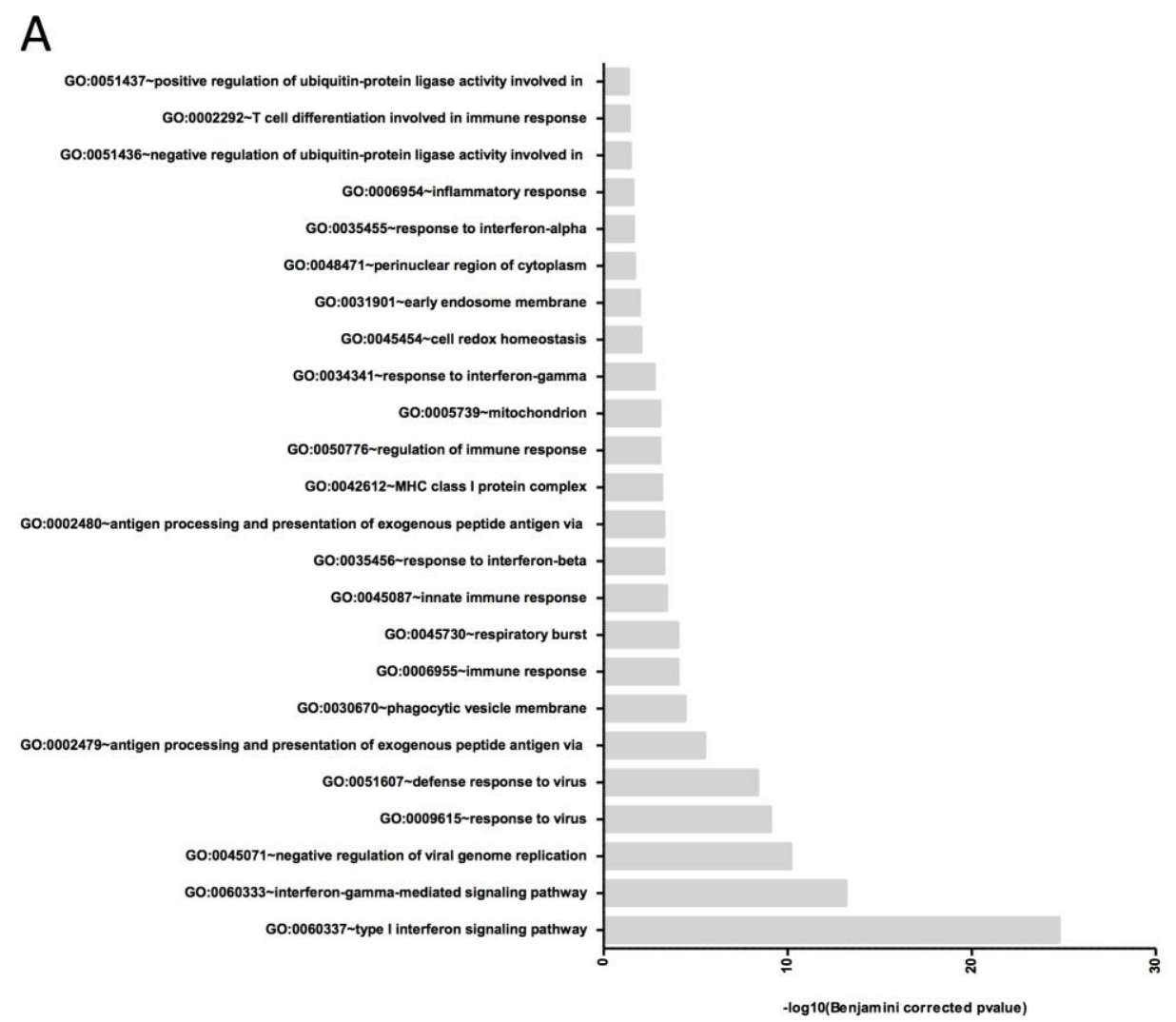

B

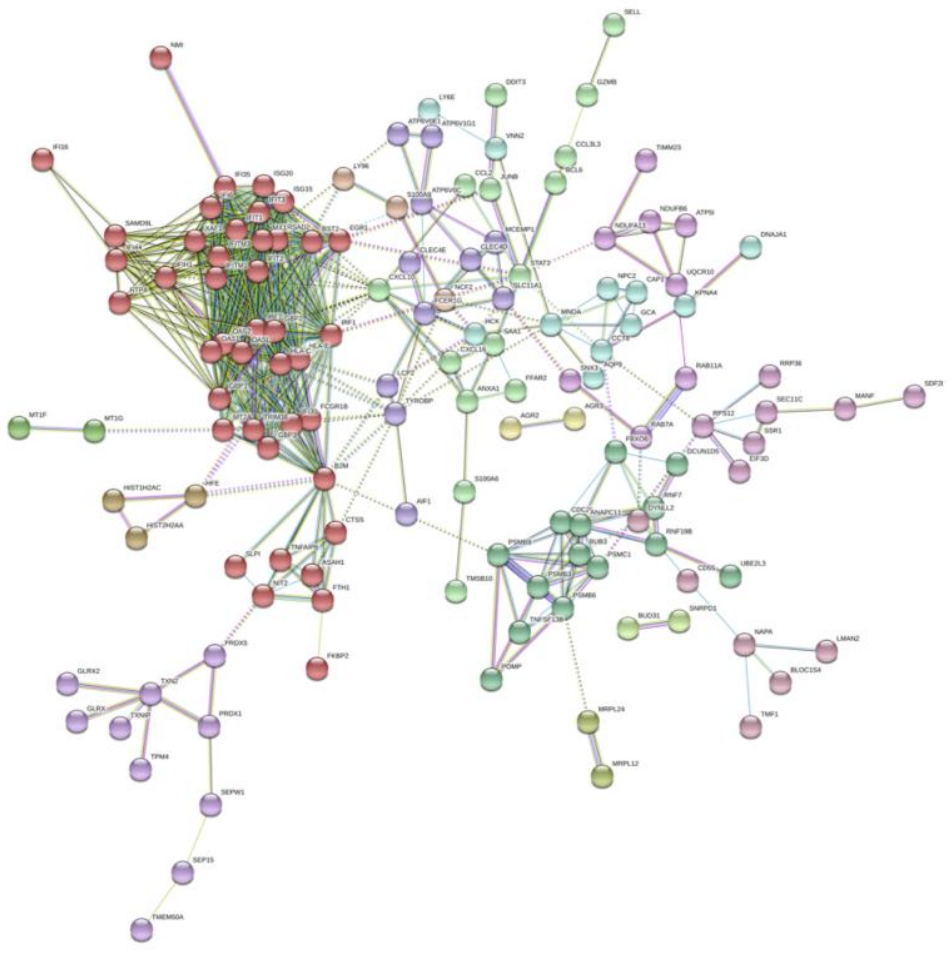


Figure 3
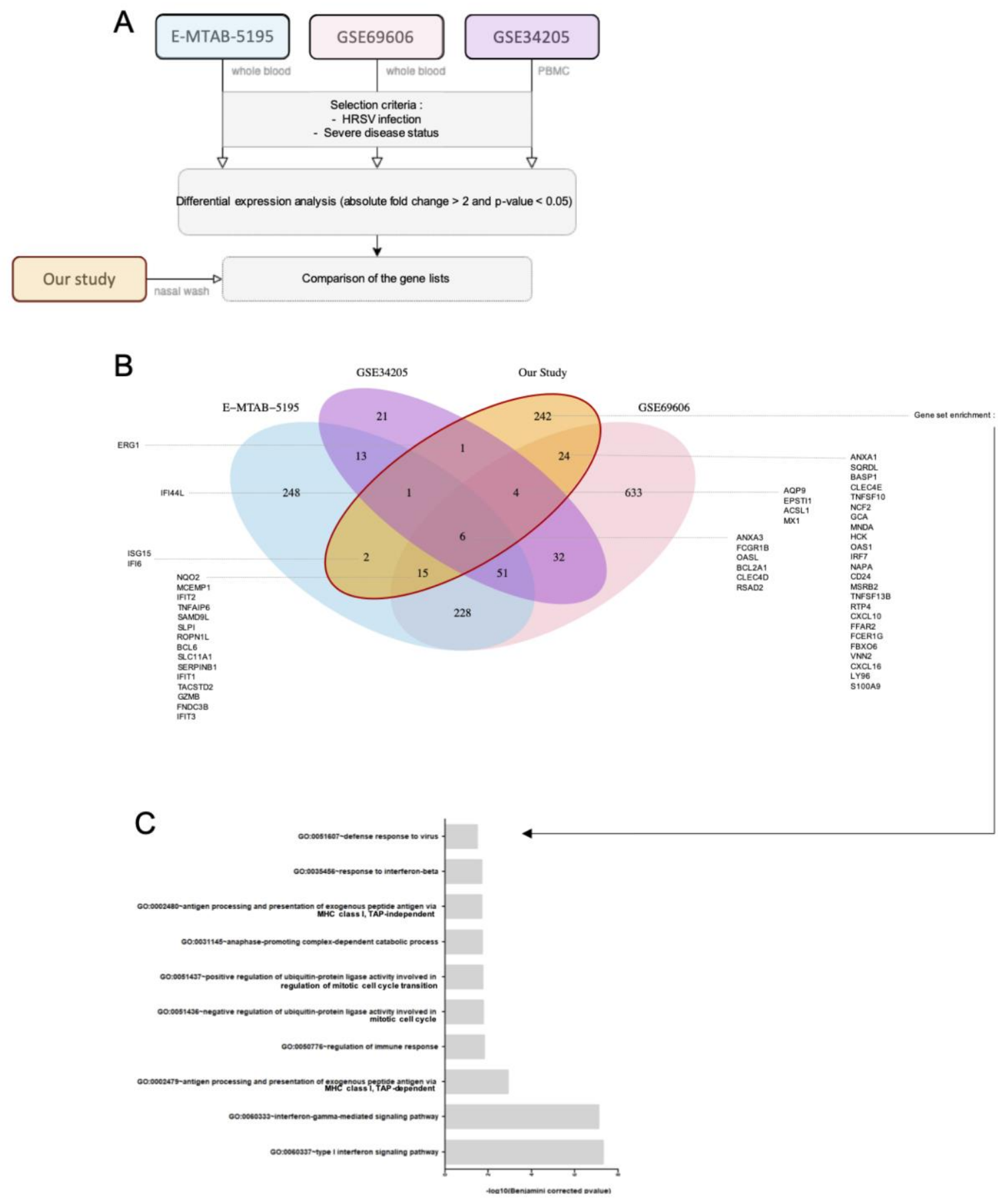
Figure 4

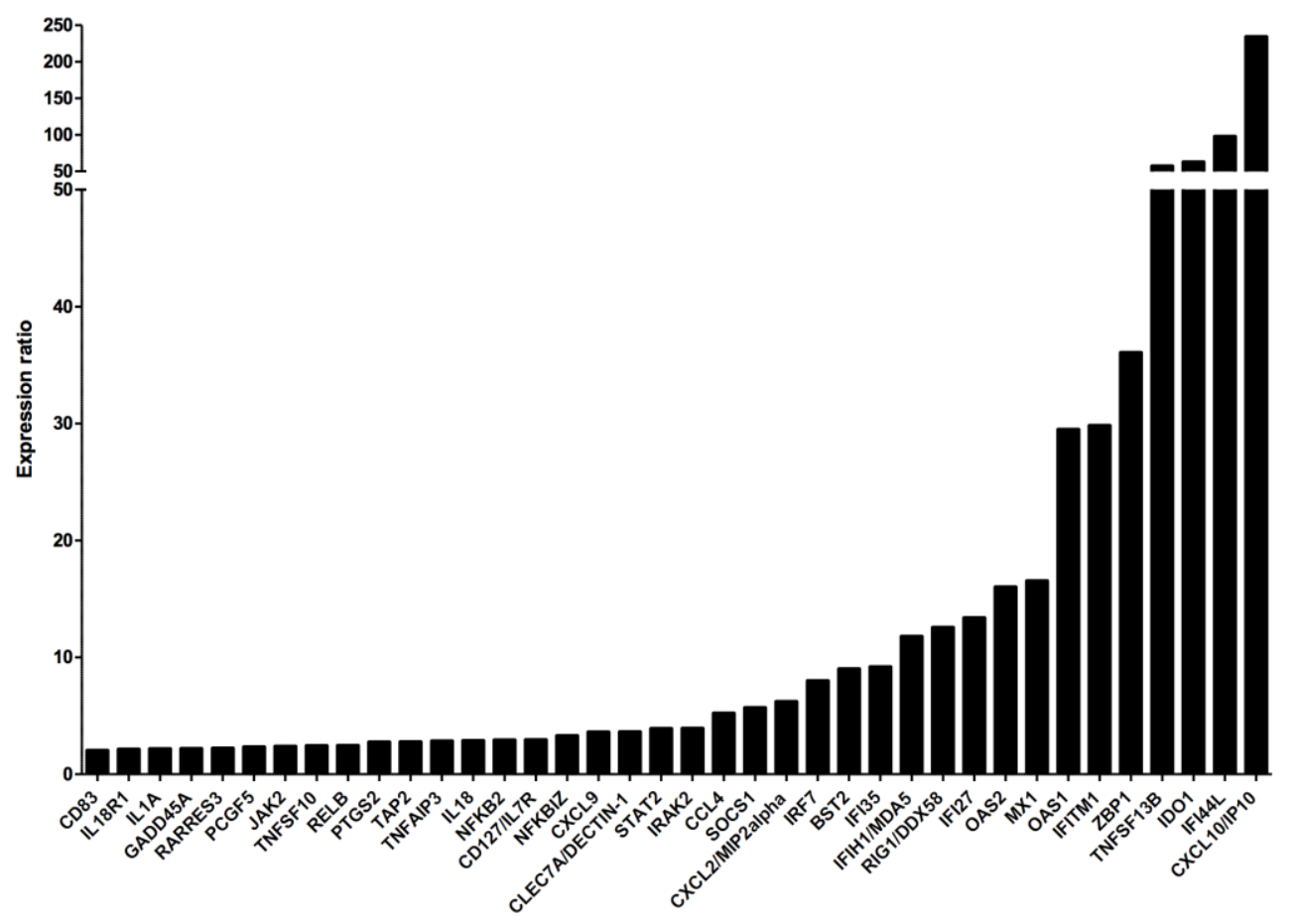

